\title{
Design of Wideband Microwave Absorbers Using Reactive Salisbury Screens with Maximum Flat Reflection
}

\author{
Gunyoung Kim · Sanghoek Kim • Bomson Lee*
}

\begin{abstract}
This paper presents a design methodology for wideband single-layered microwave absorbers with arbitrary absorption at the design center frequency using reactive Salisbury screens. The bandwidth of the absorber increases when the flatness of the reflection response at the design center frequency is maximized. Based on this observation, closed-form design formulas for wideband absorbers are derived. As they are scalable to any design frequency, wideband reactive screens can be systematically realized using two-dimensional periodic crossed-dipole structures patterned on a resistive sheet. Based on this method, a single-layered absorber with a $90 \%$ bandwidth improved to $124 \%$ of the design center frequency is presented. For the purpose of physical demonstration, an absorber with a design center frequency of $10 \mathrm{GHz}$ is designed and fabricated using a silver nanowire resistive film with a surface resistance of $30 \Omega /$ square. The measured absorption shows a good agreement with both the calculation and the electromagnetic simulation.
\end{abstract}

Key Words: Absorption Bandwidth, Design Equation, Microwave Absorber, Maximum Flatness, Reactive Salisbury Screen.

\section{INTRODUCTION}

A number of studies have developed thin and broadband absorbers that can be utilized for stealth applications and in the laboratory environment. Recent advancements in metamaterial research have opened up the possibility for metamaterial absorbers [1-3]. However, the reported bandwidth of a metamaterial absorber remains far narrower than that of conventional Salisbury absorbers.

Salisbury absorbers are some of the most representative microwave absorbers. The simplest design of Salisbury absorbers has a resistive sheet of $377 \Omega /$ square placed at a distance of a quarter wavelength from a metallic plane $[4,5]$. The bandwidth of the Salisbury screen is essentially limited by the frequency dependence of the sandwiched layer. A primitive way to en- hance the bandwidth in a Salisbury screen is simply by using more resistive sheets spaced at the same distance $(\lambda / 4)$ apart [6]. These original structures, consisting of purely resistive sheets, a dielectric slab, and a ground plane, are referred to as Jaumann absorbers. A systematic design method of Jaumann absorbers to enhance the bandwidth at the cost of a lowered absorption rate was investigated in [7].

Another popular method to widen the bandwidth is to introduce frequency-selective or reactive surfaces, the effects of which are usually modeled by simple equivalent circuits. This type of structure is usually called a circuit analog absorber [8-18]. Circuit analog absorbers were also actively studied using equivalent circuits and optimizations usually combined with multiple-layer [8, 9] multiple-resonance [10-12], or mixed [18] techniques, resulting in a significant bandwidth extension [12] up to $128 \%$ based on $90 \%$ absorption criteria. Some features of these devel-

Manuscript received June 4, 2018 ; Accepted August 20, 2018. (ID No. 20180604-047J)

Department of Electronics Engineering, Kyung Hee University, Yongin, Korea.

"Corresponding Author: Bomson Lee (e-mail: bomson@khu.ac.kr)

This is an Open-Access article distributed under the terms of the Creative Commons Attribution Non-Commercial License (http://creativecommons.org/licenses/by-nc/4.0) which permits unrestricted non-commercial use, distribution, and reproduction in any medium, provided the original work is properly cited.

(c) Copyright The Korean Institute of Electromagnetic Engineering and Science. All Rights Reserved. 
opments are as follows.

A multi-layered structure with loaded chip resistors was proposed with an enhanced $99 \%$ absorption bandwidth of about 40 $\%$ [9]. A thin and broadband radar absorber using a resistive treble-square frequency selective surface consisting of three square loops was investigated in [10]. Its relative absorption bandwidth was $92.2 \%$ at the frequency range of $10.7-29 \mathrm{GHz}$ based on a $90 \%$ absorption rate. A triple-band planar absorber with a high-impedance surface was proposed with a theoretical derivation of a matching condition requiring varied resistance as a function of frequency [11]. The reported $90 \%$ absorption bandwidth is roughly $85 \%$ of the center frequency of $10 \mathrm{GHz}$, but it requires a mixture of different unit structures having different surface resistances per square. Using resistive trumpetshaped structures coated on an FR-4 substrate, the metamaterial-inspired absorber for a wideband operation was proposed at the X-band [13]. Its $90 \%$ absorption bandwidth was $66.7 \%$ at $10.95 \mathrm{GHz}$. Clearly, by exploiting a large design space of multilayers and element patterns, the incident angle of absorption, and the bandwidth can be maximized [8].

However, multi-layer structures may not be preferred in some environments because of the limitations of the thickness and the difficulties in fabrication. Therefore, many efforts are still being made to enhance the bandwidth assuming a single-layer design [12, 14-17]. In [14], simple design equations for a wideband dipole-type absorber with complete absorption at a specific center frequency were presented, and a design example of a chipresistor-loaded absorber was demonstrated. The chip-resistorloaded absorber was later reported in $[15,19]$ as well. By employing discrete components, the thickness of the absorber can be shorter than a quarter wavelength while maintaining a relatively wide bandwidth. However, the issue of soldering many discrete components onto the circuit boards remains a drawback of this approach.

Without requiring discrete components and using a resistive silver nanowire (AgNW) film, a wideband $\mathrm{X}$-band absorber employing a cross-shaped structure was presented in [16]. Its reported $90 \%$ absorption bandwidth with complete absorption at the design frequency of $10 \mathrm{GHz}$ was about $80 \%$. In [17], a thin resistive and capacitive absorber, which was composed of resistive rectangular structures using an $\mathrm{AgNW}$ film, was analyzed and evaluated at $3 \mathrm{GHz}$. It had a wideband absorption characteristic despite its relatively thin layer (a $90 \%$ absorption bandwidth of $76 \%$ with a layer thickness of only $\lambda / 8$ at $3 \mathrm{GHz}$ ).

This work determines a systematic method to widen the bandwidth of single-layered, circuit analog absorbers without soldering discrete components. In most cases, whether one uses multi-layer structures or circuit analog absorbers, a trade-off exists between the bandwidth and the absorption at the center frequency. In other words, at the expense of a lowered absorp- tion rate, the bandwidth can increase. Although this sounds intuitively correct and has been demonstrated for Jaumann absorbers [7], the design guideline for single-layered circuit analog absorbers to balance between the bandwidth and the absorption rate has not been studied yet.

In this paper, we present simple, closed-form solutions for the design of single-layered wideband microwave absorbers with an arbitrary absorption rate at any design center frequency $\omega_{0}$. In Section II, the design equations are derived to maximize the flatness of reflection based on an equivalent circuit. Section III presents an easy guideline to finalize the dimensions of the used dipole structure without complicated optimization processes. In Section IV, a wideband microwave absorber at $10 \mathrm{GHz}$ is designed and fabricated using the AgNW resistive film. The theoretical bandwidth of the presented absorber is compared with the measured one. The conclusion is given in Section V.

\section{THEORY BASED ON AN EQUIVALENT CIRCUIT}

Fig. 1 shows the geometry of a typical microwave absorber using a reactive screen placed above a conducting plane $[16,17$, 20]. The length of the spacer $l$ is usually a quarter wavelength [4]. To realize a reactive screen, this work chooses a dipole-type structure. Specifically, a crossed-dipole pattern is chosen to make the absorption characteristics almost insensitive to the polarizations of incident electromagnetic waves, as will be seen in Section IV. The terminal impedance of the reactive screen can then be modelled as a lumped-series RLC resonator, as shown in Fig. 2. To optimize the pattern to have wideband absorption, we first analytically find the representative circuit values that should result in the maximum flatness of the reflection

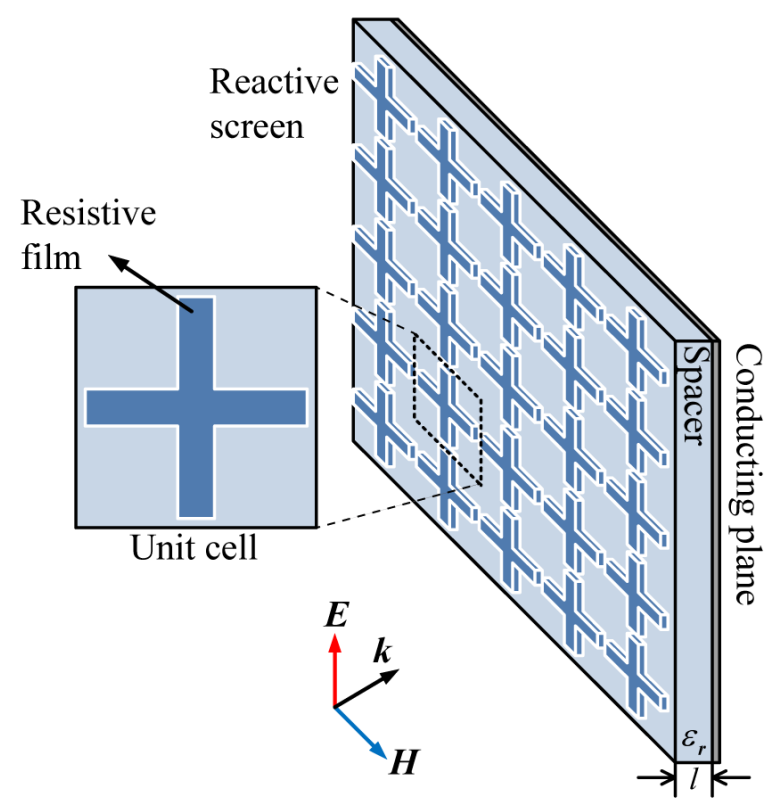

Fig. 1. Geometry of the wideband absorber with a reactive Salisbury screen. 


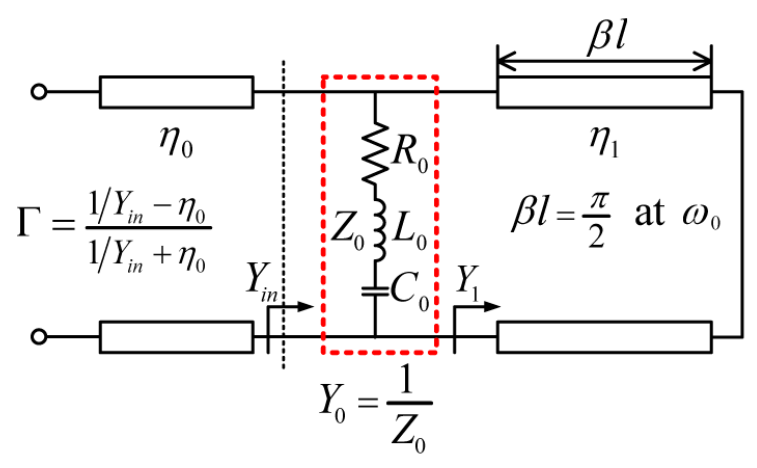

Fig. 2. Equivalent circuit of Fig. 1.

and wide bandwidth in this section. Based on the RLC values obtained from this section, the exact dimension of the pattern structure will be determined through electromagnetic (EM) simulations in Section III.

\section{Circuit Modelling of the Reactive Screen}

Fig. 2 shows the equivalent circuit composed of a series resonant circuit representing the reactive screen in the shunt branch and a quarter-wavelength transmission line that is terminated short. In Fig. 2, $\eta_{0}(=377 \Omega)$ and $\eta_{1}$ are the intrinsic impedances of free space and the spacer, respectively, and $Z_{0}$ is the impedance of the resonant circuit consisting of $R_{0}, L_{0}$, and $C_{0}$. With a quarter-wavelength spacer $l$, the electrical length $\beta l$ is $\pi / 2$ at the design center frequency $\omega_{0}$. $L_{0}$ and $C_{0}$ are chosen to resonate at the design center frequency $\omega_{0}$ in this work.

The input admittance $Y_{\text {in }}$ at the front of the screen is

$$
\begin{aligned}
& Y_{i n}(\omega)=\frac{1}{Z_{0}}+Y_{1} \\
& =\frac{1}{R_{0}+j \omega_{0} L_{0}\left(\frac{\omega}{\omega_{0}}-\frac{\omega_{0}}{\omega}\right)}-j \frac{1}{\eta_{1}} \cot \left(\frac{\pi}{2} \cdot \frac{\omega}{\omega_{0}}\right) \\
& =G_{i n}(\omega)+j B_{i n}(\omega)
\end{aligned}
$$

where $\omega_{0} L_{0}=\sqrt{L_{0} / C_{0}}$ is the reactance slope parameter of the series resonant circuit. The reflection coefficient $\Gamma$ and the absorption rate $A$ are given as

$$
\Gamma(\omega)=\frac{1 / \eta_{0}-Y_{\text {in }}(\omega)}{1 / \eta_{0}+Y_{\text {in }}(\omega)}
$$

and

$$
A(\omega)=1-|\Gamma(\omega)|^{2} .
$$

The idea of a traditional, purely resistive Salisbury screen is to use $Z_{0}=\eta_{0}=377 \Omega$, and thus match $Y_{i n}(\omega)=1 / \eta_{0}$ at the design center frequency $\omega=\omega_{0}$. This results in perfect absorption $A\left(\omega_{0}\right)$ $=A_{0}=1$ at the center frequency, but the bandwidth is relatively narrow.

\section{Design Choices for Maximum Flatness}

\subsection{Match factor}

[7] suggests that the bandwidth can be increased further by introducing a slight mismatch at the center frequency. To quantify this effect, we introduce a match factor $m$ given by

$$
R_{0}=m \eta_{0} .
$$

The absorption $A_{0}$ at the center frequency now deviates from unity as

$$
A_{0}=1-\left(\frac{m-1}{m+1}\right)^{2} .
$$

For a specifically desired absorption $A_{0}$, the match factor $m$ can either be

$$
m_{1}=\frac{1-\sqrt{1-A_{0}}}{1+\sqrt{1-A_{0}}}
$$

or

$$
m_{2}=\frac{1+\sqrt{1-A_{0}}}{1-\sqrt{1-A_{0}}} .
$$

When the required absorption $A_{0}$ is unity, we have $m_{1}=m_{2}=$ 1. However, when a slight mismatch is introduced, we have two options to determine $R_{0} ; m_{1}<1$ and $m_{2}>1$.

\subsection{Choice of $L_{0}$ and $C_{0}$ for maximum flatness}

$L_{0}$ and $C_{0}$ can be determined to enhance the bandwidth. Reminiscent of how the Butterworth filter has a maximum flat response [21], the bandwidth can be widened by enforcing

$$
\left.\frac{d^{n}|\Gamma(\omega)|^{2}}{d \omega^{n}}\right|_{\omega=\omega_{0}}=0
$$

for as large an $n$ as possible. Intuitively, if (8) is satisfied for every non-negative integer $n$, $\Gamma$ is simply zero for an infinite bandwidth. In this work, we have only one independent energy storage element $L_{0}$. Once $L_{0}$ is determined, $C_{0}$ is determined accordingly to resonate with $L_{0}$ at the design center frequency $\omega_{0}$. With one independent energy storage element, the number of derivatives that can be set to zero in (8) is at most one in general. The condition of $\left.\frac{d|\Gamma(\omega)|^{2}}{d \omega}\right|_{\omega=\omega_{0}}=0$ can be readily fulfilled by enforcing

$$
\left.\frac{d Y_{i n}(\omega)}{d \omega}\right|_{\omega=\omega_{0}}=0 .
$$

Moreover, if (9) is satisfied, as $|\Gamma(\omega)|$ must be close to zero in 
any practical absorber design, the second derivative in (8) will automatically be largely satisfied (refer to the Appendix for the derivation). This assures the design to have the maximum flatness of the response with a limited number of independent energy storage elements.

Certainly, the maximum flatness of the reflection at the design center frequency does not necessarily imply maximum bandwidth for arbitrary criteria, such as $99 \%$ or $90 \%$. Intuitively, the maximum flatness condition is more similar to the maximum bandwidth condition for wideband absorption, such as 99 \% bandwidth, than that to low ones. Nevertheless, regardless of specific bandwidth criteria, this is a good starting point before further optimization. Therefore, for wideband absorption, we first look for the values of $L_{0}$ and $C_{0}$ that yield maximum flatness, satisfying (9).

Eq. (9) implies that the derivative of both the input conductance $G_{i n}$ and the input susceptance $B_{\text {in }}$ should be zero at $\omega=\omega_{0}$. From the expression in (1), the derivative of the input conductance $G_{\text {in }}$ is zero at $\omega=\omega_{0}$. Therefore, the requirements for $L_{0}$ and $C_{0}$ are obtained from the condition $\left.\frac{d B_{i i n}(\omega)}{d \omega}\right|_{\omega=\omega_{0}}=0$.

The derivative of $B_{\text {in }}$ is given by

$$
\begin{aligned}
& \frac{d B_{i n}(\omega)}{d \omega}= \\
& -L_{0}\left[1+\frac{\omega_{0}^{2}}{\omega^{2}}\right] \frac{\left(m \eta_{0}\right)^{2}-\left(\omega_{0} L_{0}\right)^{2}\left(\frac{\omega}{\omega_{0}}-\frac{\omega_{0}}{\omega}\right)^{2}}{\left.\left(m \eta_{0}\right)^{2}+\left(\omega_{0} L_{0}\right)^{2}\left(\frac{\omega}{\omega_{0}}-\frac{\omega_{0}}{\omega}\right)^{2}\right]^{2}} \\
& +\frac{\pi}{2 \eta_{1} \omega_{0}} \csc ^{2}\left(\frac{\pi}{2} \cdot \frac{\omega}{\omega_{0}}\right) .
\end{aligned}
$$

Equating (10) to zero at $\omega=\omega_{0}$ yields $L_{0}$ and $C_{0}$ as

$$
L_{0}=\frac{\left(m \eta_{0}\right)^{2}}{\eta_{1}} \cdot \frac{\pi}{4 \omega_{0}}
$$

and

$$
C_{0}=\frac{1}{\omega_{0}^{2} L_{0}}=\frac{\eta_{1}}{\left(m \eta_{0}\right)^{2}} \cdot \frac{4}{\pi \omega_{0}}
$$

the values of which are determined according to a desired match factor $m$.

\subsection{Choice of match factor}

To investigate which match factor among $m_{1}$ and $m_{2}$ is a better choice, the circuit simulation of $B_{\text {in }}$ in Fig. 2 is run, and the results are compared.

When $A_{0}=0.9$ for example, the match factor can either be $m_{1}=0.5195$ or $m_{2}=1.925$. For each case, we have an $\left(R_{0}, \omega_{0} L_{0}\right)$ of $(196 \Omega, 80 \Omega)$ and $(725.2 \Omega, 1,096.4 \Omega)$, respectively. Fig. 3 shows the susceptances of the reactive screen $B_{0}$, shortterminated quarter-wavelength transmission line $B_{1}$, and their sum $B_{\text {in }}$ for these two cases. Clearly, for both cases, the susceptance and its slope are zero at the design center frequency, as expected.

Conversely, the choice of $m_{1}$ in Fig. 3(a) leads to a wider resonant bandwidth than the choice of $m_{2}$ in Fig. 3(b). The reason is that the two zero-derivatives of $B_{0}$ are farther apart with $m_{1}$ than with $m_{2}$. For example, in Fig. 3 , the distance between the two zero-derivatives of $B_{0}$ is about $1.5 \omega_{0}\left(=1.9 \omega_{0}-0.4 \omega_{0}\right)$ for $m_{1}$, which is much greater than $0.45 \omega_{0}\left(=1.3 \omega_{0}-0.85 \omega_{0}\right)$ for $m_{2}$. From (10), it is straightforward to show that this holds generally true for any choice of absorption $A_{0}$; i.e., the zero-

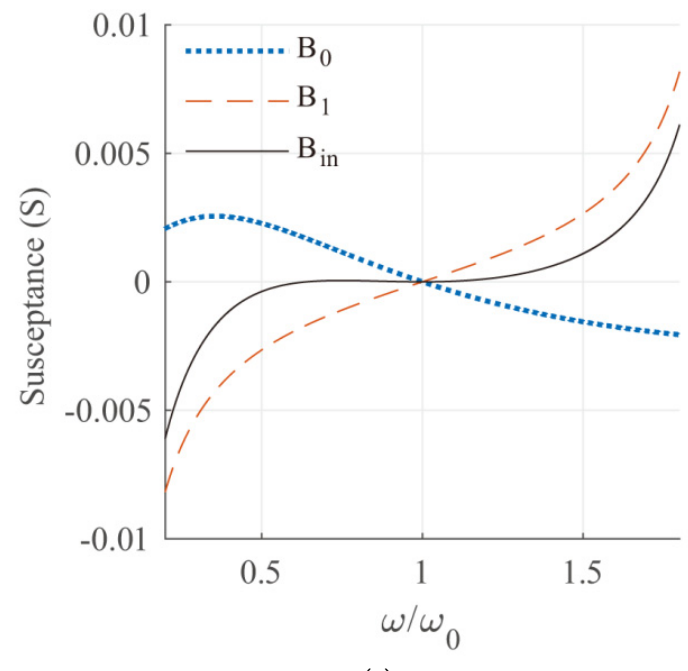

(a)

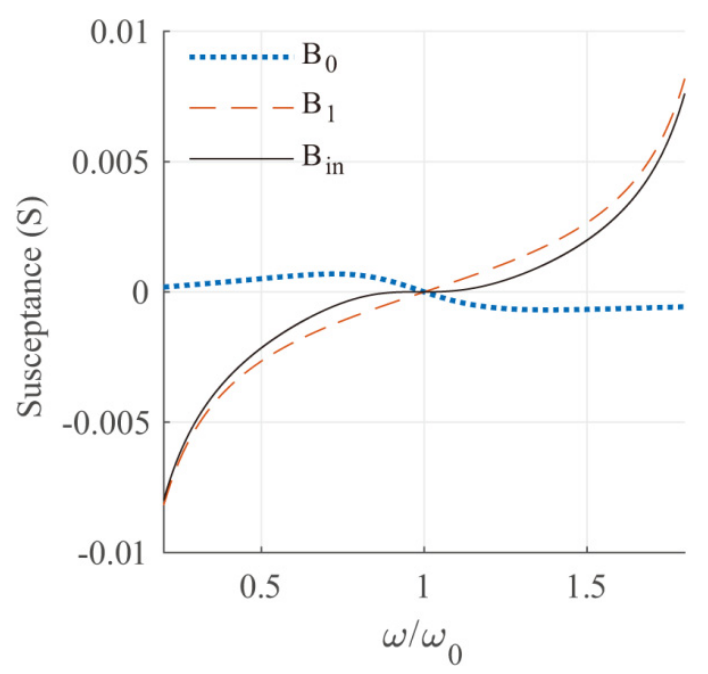

(b)

Fig. 3. Choice of match factor. Susceptances of the reactive screen $B_{0}$, short-terminated quarter-wavelength transmission line $B_{1}$, and their sum $B_{\text {in }}=B_{0}+B_{1}$ when $A_{0}=0.9$ as a function of normalized frequency for the cases of (a) $m_{1}=0.5195$ and (b) $m_{2}=1.925$. 
derivatives of $B_{0}$ are split farther apart for smaller match factors. The wide resonant bandwidth of $B_{\text {in }}$ naturally broadens the absorption bandwidth. Therefore, in this work, the smaller match factor $m_{1}$ of (6) is chosen to determine $R_{0}, L_{0}$, and $C_{0}$ in an effort to enhance the absorption bandwidth.

\section{Final Design Adjustment through Circuit Simulation}

We now evaluate the effect of the maximum flatness design on the absorption bandwidth. Fig. 4(a)-(b) show the input impedance and absorption as a function of the normalized frequency obtained from the Agilent ADS circuit simulation in Fig. 2. The results of the purely resistive Salisbury screen and those of the proposed reactive Salisbury screens with $A_{0}=1$ and 0.9 are compared. For simplicity, the relative permittivity of the spacer layer is assumed to be unity. The used circuit components $\left(R_{0}, \omega_{0} L_{0}\right)$ of the resistive Salisbury screen, the reactive one with $A_{0}=1$, and the reactive one with $A_{0}=0.9$ are (377 $\Omega$, $0 \Omega$ ), (377 $\Omega, 296 \Omega$ ), and $(196 \Omega, 80 \Omega$ ), respectively.

In Fig. 4(a), the reactance of the reactive absorbers is zero over a wide bandwidth region near the design center frequency. This property, which the purely resistive Salisbury screen does not possess, endows the absorber with a much wider bandwidth, as shown in Fig. 4(b). Furthermore, a wider flatness both in the resistance and the reactance near the design frequency is observed when sacrificing absorption at the center frequency from $A_{0}=1$ to $A_{0}=0.9$, as shown in Fig. 4(a). As a result, when $A_{0}=$ 0.9 , the $90 \%$ absorption bandwidth reaches up to $119 \%$ from

\section{$0.35 \omega_{0}$ to $1.54 \omega_{0}$ (Fig. $\left.4(\mathrm{~b})\right)$.}

If necessary, the adjustments for $L_{0}$ and $C_{0}$ can be made to further enhance the bandwidth according to specific bandwidth criteria. For example, let us assume that we are interested in maximizing the $90 \%$ absorption bandwidth. In Fig. 4(b), the maximum flatness design of $A_{0}=0.9$ suffers asymmetry around the design center frequency $\omega_{0}$. This scenario is better visualized using the Smith chart in Fig. 4(c). The reflection coefficient $\Gamma$ of the maximum flatness design quickly moves to the short impedance above the design center frequency. This results in the absorption bandwidth being considerably narrow in the upper range, $\omega_{0}-1.54 \omega_{0}$, compared with $0.35 \omega_{0}-\omega_{0}$ in the lower range.

An effective way to increase the upper bandwidth is to slightly increase the inductive reactance $\omega_{0} L_{0}$, creating an additional resonant frequency beyond the design center frequency, as shown in the solid curve in Fig. 4(c). The appearance of an additional resonant frequency is not a coincidence. The maximum flatness design places multiple roots of the resonance at the design center frequency $\omega_{0}$ because $B_{i n}\left(\omega_{0}\right)=\left.\frac{d}{d \omega} B_{i n}(\omega)\right|_{\omega=\omega_{0}}=0$. Therefore, a slight change in parameters from the maximum flatness necessarily results in split resonances. Certainly, this split can further increase the bandwidth. For example, the inductive reactance $\omega_{0} L_{0}$ is chosen to be $120 \Omega$ to further increase the $90 \%$ absorption bandwidth up to $124 \%$ (Fig. 5). The capacitance $C_{0}$ is again determined accordingly by $\omega_{0}=1 / \sqrt{L_{0} C_{0}}$. The circuit parameters and the resultant $90 \%$ absorption bandwidth

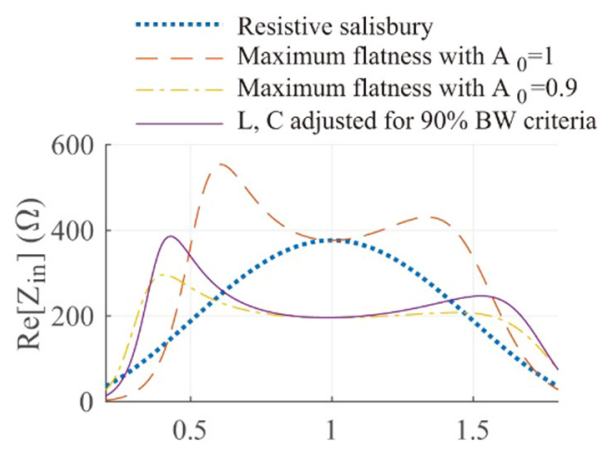

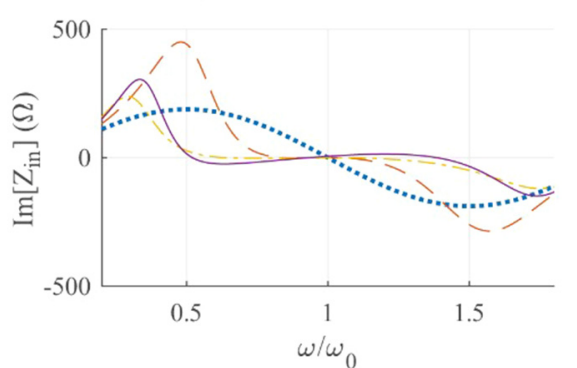

(a)

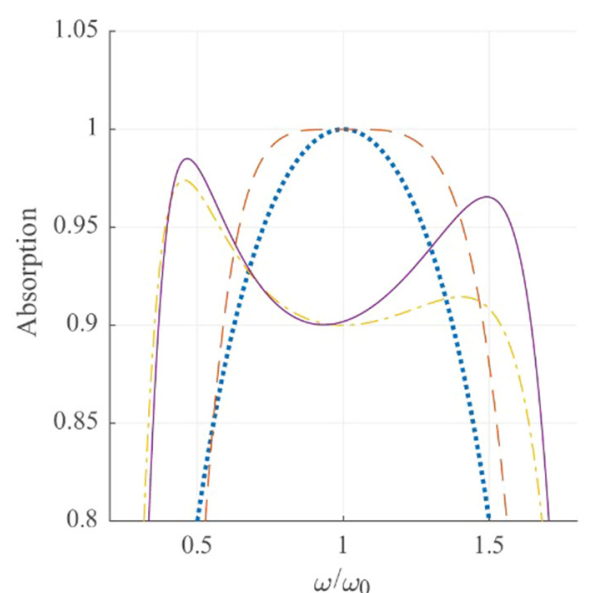

(b)

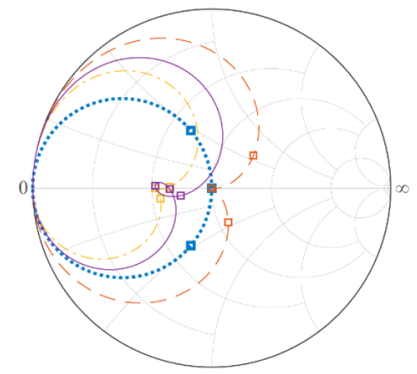

(c)

Fig. 4. (a) Input impedances and (b) absorption of the air-spaced absorber as a function of normalized frequency. The $\left(R_{0}, \omega_{0} L_{0}\right)$ for each absorber are as follows: resistive Salisbury $(377 \Omega, 0 \Omega)$, maximum flatness with $A_{0}=1$ : $(377 \Omega, 296 \Omega)$, maximum flatness with $A_{0}$ $=0.9(196 \Omega, 80 \Omega)$, and final adjustment for $90 \%$ criteria $(196 \Omega, 120 \Omega)$. (c) The Smith chart showing the reflection coefficients of each design. All of the traces rotate clockwise with the frequency. The markers on the traces represent the frequencies of $0.6 \omega_{0}$, $\omega_{0}$, and $1.4 \omega_{0}$ in order. 


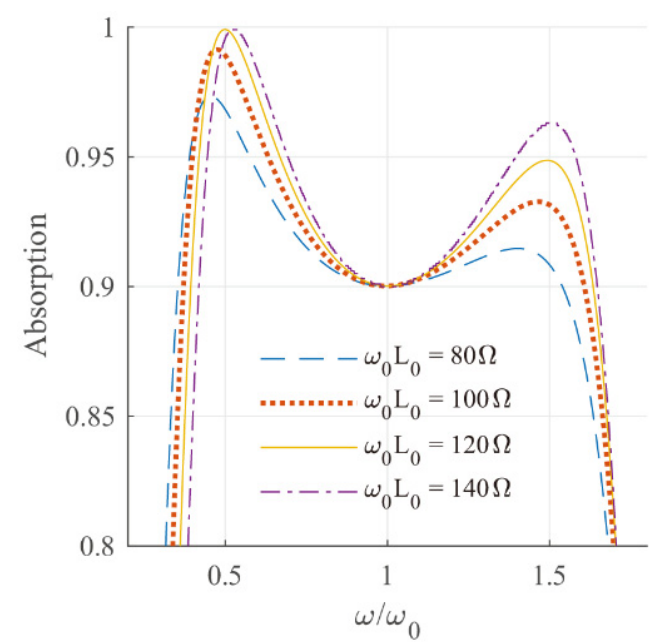

Fig. 5. Absorption bandwidth with varying inductive reactance $\omega_{0} L_{0}$. The $90 \%$ bandwidth is $119 \%, 123 \%, 124 \%$, and $123 \%$ when $\omega_{0} L_{0}$ is $80 \Omega, 100 \Omega, 120 \Omega$, and $140 \Omega$, respectively.

are presented in Table 1.

Let us summarize the method of obtaining the required circuit components representing the reactive screen. If a specific $A_{0}$ is desired at $\omega_{0}$, the match factor $m_{1}<1$ is obtained using (6). Then $R_{0}, L_{0}$, and $C_{0}$ are first determined to maximize the flatness of the reflection at the design center frequency following (4), (11), and (12). The maximum flatness essentially widens the bandwidth of the absorption. To increase the bandwidth specifically for certain criteria, $L_{0}$ and $C_{0}$ can be tuned to maximize the bandwidth through circuit simulation while maintaining $\omega_{0}=1 / \sqrt{L_{0} C_{0}}$.

\section{CROSSED-Dipole STRUCTURE TO SyNTHESIZE THE CIRCUIT PARAMETERS}

The design procedures explained in Section II are scalable to any frequency in general. In this work, we build an absorber around the center frequency of $10 \mathrm{GHz}$.

We physically realize the circuit parameters obtained in Section II with the crossed-dipole structure. In Fig. 6(a), $a$ is the side length of the absorber square unit, $w$ and $b$ are the width and height of the crossed-dipole made of a resistive film, respectively, $R_{s}$ is the resistance per square of the film material, and $g$ is the gap distance.

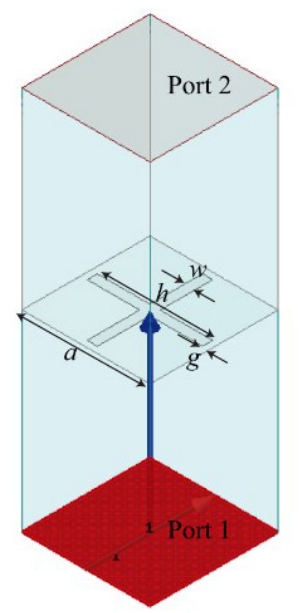

(a)

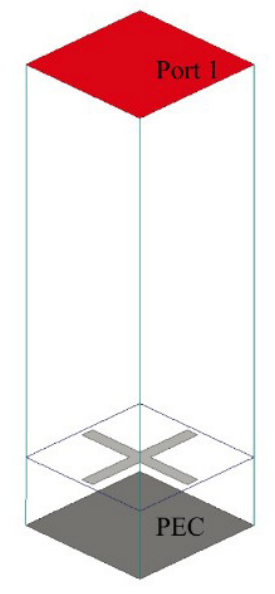

(b)
Fig. 6. Electromagnetic simulation setup to evaluate terminal impedance (a) and absorption (b).

The terminal impedance on the resistive film is a function of the structure dimension. Clearly, the series circuit components $R_{0}, L_{0}$, and $C_{0}$ of the unit cell are monotonous functions of $R_{s} b / w, b / w$, and $w / g$, respectively. Therefore, finding a dimension to synthesize the required circuit components requires only a few iterations of electromagnetic simulations. In other words, by separating the work of optimization and realization each done by the circuit and electromagnetic simulations, the design process of the structure is less expensive in terms of computation than other optimization approaches, which heavily rely on electromagnetic simulations $[13,19,20]$.

To evaluate the terminal impedance, a two-port simulation is run in Ansys HFSS with de-embedding of the effect of finite distances between the ports and the film, as shown in Fig. 6(a). Through this simulation, the dimensions of the unit structure that realize the required circuits values in Table 1 are found and tabulated in the same table.

The simulated absorption using HFSS is also straightforward, as shown in Fig. 6(b). A perfect electric conductor is placed at a quarter-wavelength distance behind the pattern. For example, when an air medium is used as a spacer, the absorption of the crossed-dipole structures in Table 1 is simulated in HFSS and compared with those of the corresponding circuits in Fig. 7. As the terminal impedance is well synthesized by the pattern structure (although not included in this work), the absorption of the

Table 1. Circuit parameters and their $90 \%$ absorption bandwidths from circuit simulation

\begin{tabular}{lcccccccc}
\hline \multicolumn{1}{c}{$A_{0}$} & $R_{0}(\Omega)$ & $\omega_{0} L_{0}(\Omega)$ & $\mathrm{BW}(\%)$ & Remarks & $R_{s}(\Omega / \square)$ & $a(\mathrm{~mm})$ & $w(\mathrm{~mm})$ & $h(\mathrm{~mm})$ \\
\hline 1 & 377 & 296 & 89 & Maximum flatness & 30 & 10 & 0.8 & 9.8 \\
0.99 & 308 & 198 & 105 & Maximum flatness & 30 & 8.7 & 0.8 & 8.6 \\
0.9 & 196 & 80 & 119 & Maximum flatness & 50 & 8.5 & 2.2 & 8.4 \\
0.9 & 196 & 120 & 124 & $L, C$ adjusted for 90\% BW criteria & 40 & 8.6 & 1.7 & 8.5 \\
\hline
\end{tabular}

The dimensions of the crossed-dipole structure are also shown. 


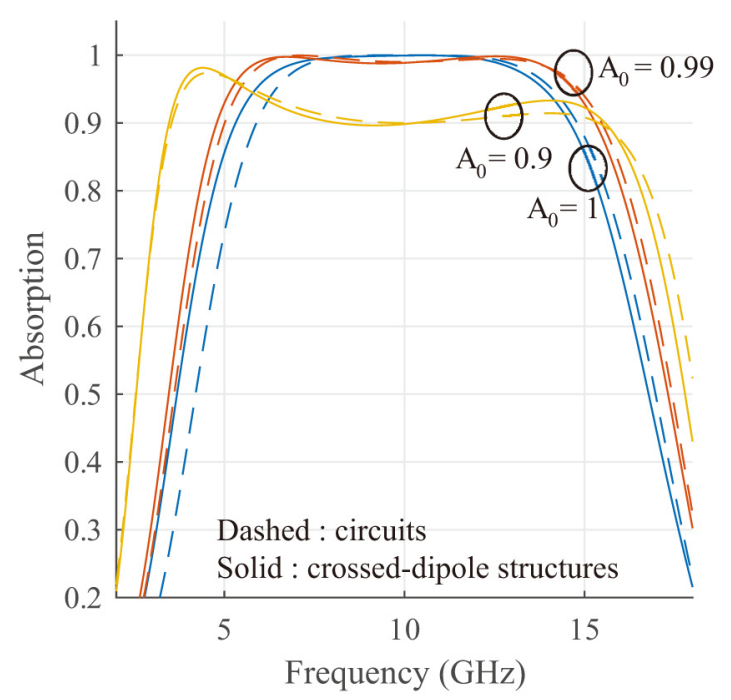

Fig. 7. Absorption of air-spaced maximum flatness design: circuit (dashed line) and electromagnetic (solid line) simulations.

Table 2. Bandwidth and thickness comparison of the reported single-layer absorber structures

\begin{tabular}{cccc}
\hline Ref. & $\begin{array}{c}\text { Bandwidth } \\
(\%)\end{array}$ & $\begin{array}{c}\text { Thickness } \\
\left(\lambda_{0}\right)\end{array}$ & Unit cell \\
\hline$[12]$ & 98.4 & 0.25 & Resistive square patch \\
{$[22]$} & 81.7 & 0.13 & Hexagonal with resistors \\
{$[15]$} & 70.7 & 0.12 & Crossed-dipole with resistors \\
{$[16]$} & 80 & 0.25 & Crossed-shaped resistive film \\
{$[17]$} & 76 & 0.125 & Square-shaped resistive film \\
{$[20]$} & 117 & 0.2 & Hexagonal with film surface \\
{$[19]$} & 105 & 0.128 & Dipole antenna with resistors \\
This work & 124 & 0.25 & Crossed-dipole resistive film \\
\hline
\end{tabular}

maximum flatness designs from HFSS successfully reproduces the circuit behaviors.

Additionally, a comparison between the absorber proposed here and those reported in the literatures is presented in Table 2. Our design methodology based on the maximum flatness of the equivalent circuit design is mathematically straightforward and requires neither many iterative electromagnetic simulations nor the soldering of external components. Nevertheless, it provides quality performance in terms of the bandwidth compared with other published works.

\section{FABRICATION AND MEASUREMENT}

For the purpose of demonstration, the crossed-dipole absorber with the required absorption of $A_{0}=1$ at the design center frequency of $10 \mathrm{GHz}$ is fabricated and measured.

\section{Design and Simulation}

The AgNW film with $30 \Omega /$ square is used for a resistive sheet. AgNWs are chosen for fabrication because their sheet resistance can be easily controlled by adjusting the concentration of the AgNW solution [23]. Fig. 8 shows the geometry of the unit cell for the fabricated absorber. Owing to the difficulty of applying and laser-cutting the film directly on to a Styrofoam spacer with $\varepsilon_{r}=1.03$, we first attached the film on an acrylic layer with $\varepsilon_{r}=2.56$, which was later mounted on the Styrofoam layer. The acrylic layer with a thickness $l_{1}$ of $1.5 \mathrm{~mm}$ was used. The thickness of the Styrofoam $l_{2}$ was determined to be $4 \mathrm{~mm}$ to ensure a zero susceptance of $Y_{1}$ at $10 \mathrm{GHz}$. Based on the same condition, the effective permittivity $\boldsymbol{\varepsilon}_{e f f}$ and the intrinsic impedance $\eta_{1}$ of an effective single-layer spacer were 1.86 and $276.5 \Omega$, respectively.

With $m=1, \eta_{0}=377 \Omega$, and $\eta_{1}=276.5 \Omega$, the circuit values for the maximum flatness were $\left(R_{0}, L_{0}, C_{0}\right)=(377 \Omega, 6.42 \mathrm{nH}$, $39.5 \mathrm{fF})$. The fine adjustments of $L_{0}$ and $C_{0}$ for further bandwidth enhancement were omitted in this fabrication example. After all, the dimensions of the unit structure to realize the above circuit values were determined through electromagnetic simulations and are summarized in Table 3.

The input impedance $Z_{\text {in }}$ of the structure in Table 3 was first simulated using HFSS in Fig. 9(a) and then compared with the

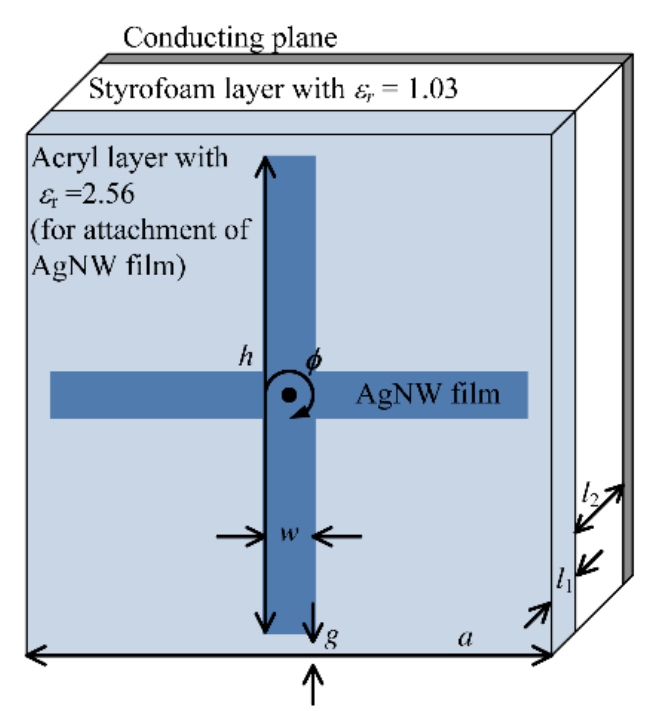

Fig. 8. Geometry of a unit structure for the fabricated absorber employing a crossed-dipole structure.

Table 3. Dimensions of the fabricated unit structure

\begin{tabular}{lc}
\hline \multicolumn{1}{r}{ Parameter } & Value \\
\hline$R_{s}(\Omega / \square)$ & 30 \\
$a(\mathrm{~mm})$ & $10.8\left(0.36 \lambda_{0}\right)$ \\
$l_{1}(\mathrm{~mm})$ & $1.5\left(0.05 \lambda_{0}\right)$ \\
$l_{2}(\mathrm{~mm})$ & $4\left(0.13 \lambda_{0}\right)$ \\
$w(\mathrm{~mm})$ & $1\left(0.03 \lambda_{0}\right)$ \\
$h(\mathrm{~mm})$ & $10\left(0.33 \lambda_{0}\right)$ \\
\hline
\end{tabular}




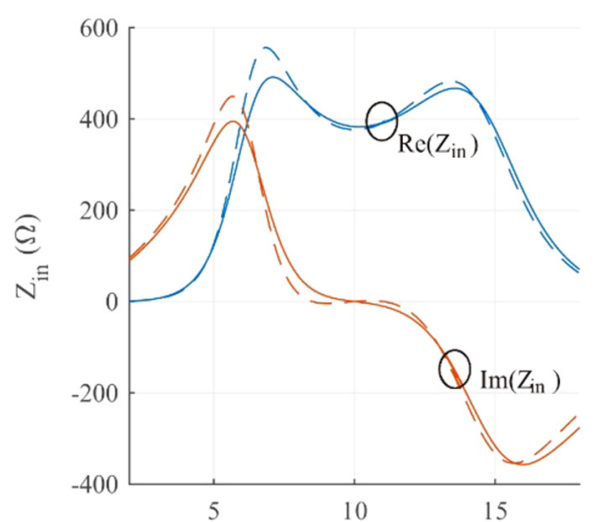

(a)

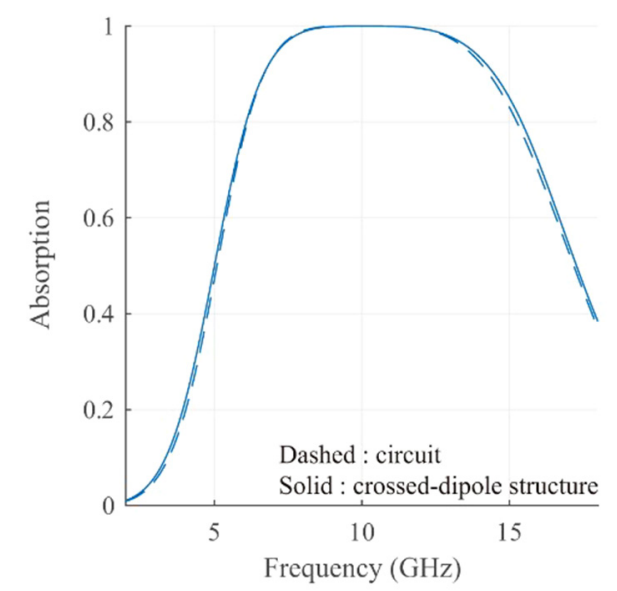

(b)

Fig. 9. (a) Input impedance and (b) absorption of a unit of the reactive screen employing a crossed-dipole structure when $A_{0}=$ 1 (solid lines). They synthesize the circuits with a maximum flatness impedance $\left(R_{0}, L_{0}, C_{0}\right)=(377 \Omega, 6.42 \mathrm{nH}$, $39.5 \mathrm{fF}$ ) (dashed lines).

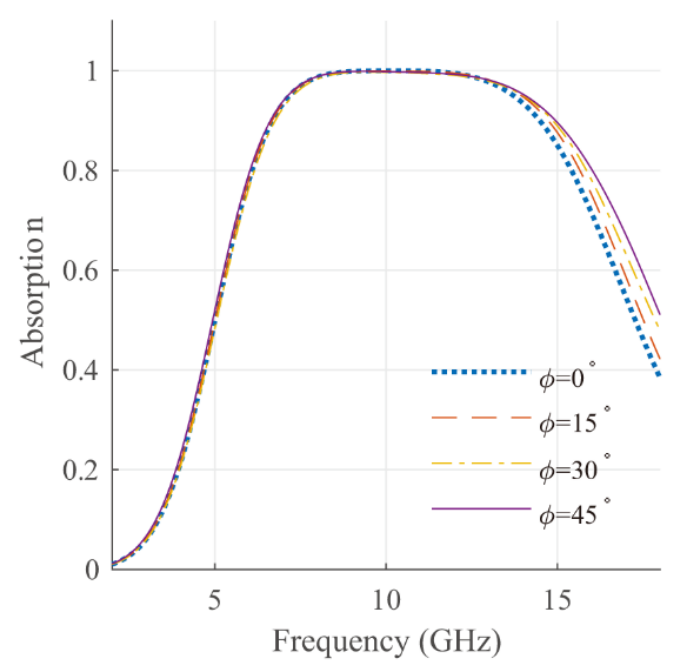

Fig. 10. Absorptions of the proposed absorber depending on polarization angles.

input impedance of the circuits in Fig. 2 with $\left(R_{0}, L_{0}, C_{0}\right)=$ $(377 \Omega, 6.42 \mathrm{nH}, 39.5 \mathrm{fF})$ for the maximum flatness. The crossed-dipole structure realized the impedances for the maximum flatness across a wide frequency range. Therefore, the absorption of the crossed-dipole structure was also in good agreement with that of the circuit in Fig. 9(b), showing the wide bandwidth. The EM-simulated $99 \%$ and $90 \%$ absorption bandwidths were about $42.7 \%$ and $78.1 \%$, respectively.

We also considered a normal incidence case with different polarization angles $\phi$. Fig. 10 shows the electromagnetically simulated absorptions depending on the polarization angles of the normally incident electric fields from $0^{\circ}$ to $45^{\circ}$. The polarization angle exhibited negligible effects on the absorption due to the employed crossed-dipole structure. For the degradation effects with oblique TE and TM incidence cases, we can apply similar techniques given in [8], but these are not included in this work.

\section{Fabrication and Measurement}

Fig. 11(a) shows the photograph of the fabricated absorber, which was made of the AgNW resistive film, acryl, Styrofoam, and conducting plane. The absorber consisted of $25 \times 17$ units, and the overall size was $270.5 \mathrm{~mm} \times 183.9 \mathrm{~mm} \times 4.5 \mathrm{~mm}$. The experimental setup is illustrated in Fig. 11(b). The absorption was measured by the horn antenna of Model 3117 (1-18 $\mathrm{GHz}$ ) produced by ETS Lindgren Inc.

Fig. 12 shows the circuit simulation, the EM simulation, and the measurement of the absorption of the proposed screen with a design center frequency of $10 \mathrm{GHz}$. The measured absorption

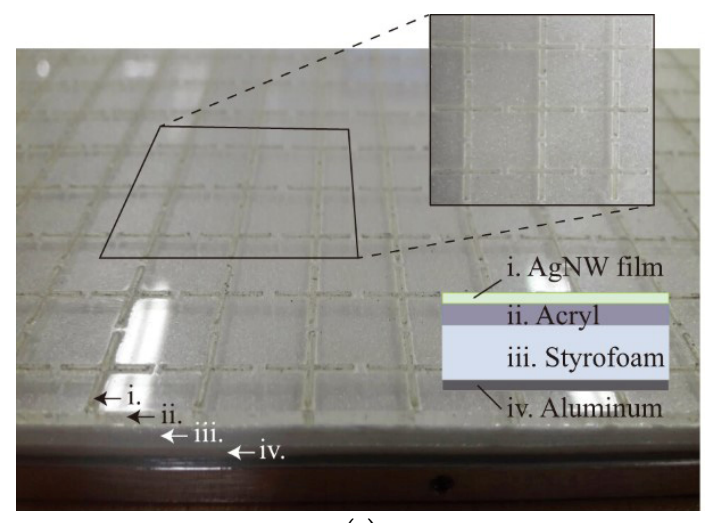

(a)

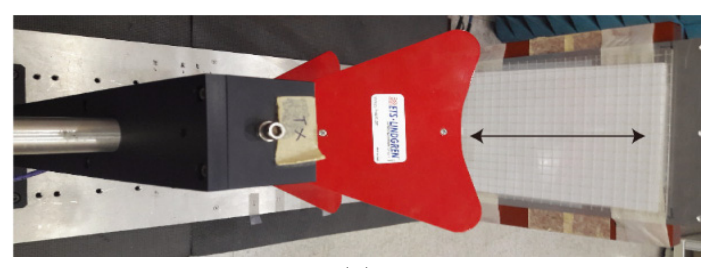

(b)

Fig. 11. Photograph of (a) the fabricated proposed reactive screen realized on an acrylic layer and (b) the experimental setup. The inset of (a) shows the closed view of the crossed-dipole structure. 


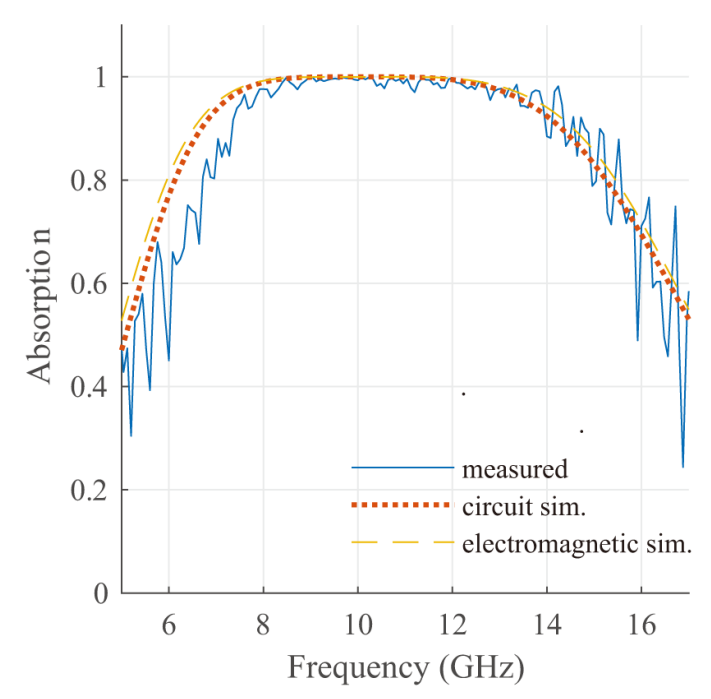

Fig. 12. Absorption of the proposed absorber.

was obtained by estimating $\left|S_{11}\right|^{2}$ on the absorber plane based on the reflection coefficient measured by the horn antenna. In the measurement, only the reflection on the absorber plane was considered, not the horn antenna itself. To calibrate the selfreflection of the horn antenna, the horn antenna in free space is used as the match load in the calibration process instead of the typical $50 \Omega$ load. In addition, the reflection coefficient in front of the reversed absorber with the same size as the absorber is used as the reference to compensate for the energy leakage through the space. A more detailed measurement method using a single antenna can be referenced in [13]. For example, the $90 \%$ absorption bandwidths of each one are $75.7 \%, 78.1 \%$, and $66.4 \%$, respectively. The difference between the simulation and the measurement could be attributed to the non-uniformity of the resistivity in the AgNW film and the limited size of the absorber in the measurement.

\section{CONCLUSION}

Based on the requirements of a maximum flat reflection at a design center frequency, a simple closed-form design solution of a reactive Salisbury screen has been derived. Having maximum flatness widens the bandwidth. For example, for the desired absorptions of $100 \%, 99 \%$, and $90 \%$ at a design center frequency, the $90 \%$ bandwidths can be widened to $89 \%, 105 \%$, and $119 \%$, respectively, from $74 \%$ for a traditional Salisbury screen. Furthermore, to maximize the bandwidth with specific criteria, further numerical optimization can be made by tuning the circuit parameters, starting from the maximum flatness condition.

For fabrication and measurement, an absorber with a design center frequency of $10 \mathrm{GHz}$ was designed and fabricated using the AgNW resistive film with a surface resistance of 30 $\Omega /$ square. The measured absorption showed a good agreement with the simulation results, validating the effectiveness of the proposed design procedures.

This work was supported by the Institute for Information \& Communications Technology Promotion (IITP) grant funded by the Korea government (MSIT) (No. IITP-20182016-0-00291, Information Technology Research Center).

\section{REFERENCES}

[1] H. Li, L. H. Yuan, B. Zhou, X. P. Shen, Q. Cheng, and T. J. Cui, "Ultrathin multiband gigahertz metamaterial absorbers," Journal of Applied Physics, vol. 110, article no. 014909, 2011.

[2] J. Lee, M. Yoo, and S. Lim, "A study of ultra-thin single layer frequency selective surface microwave absorbers with three different bandwidths using double resonance," IEEE Transactions on Antennas and Propagation, vol. 63, no. 1, pp. 221-230, 2015.

[3] F. Costa, S. Genovesi, A. Monorchio, and G. Manara, "A circuit-based model for the interpretation of perfect metamaterial absorbers," IEEE Transactions on Antennas and Propagation, vol. 61, no. 3, pp. 1201-1209, 2013.

[4] W. W. Salisbury, "Absorbent body for electromagnetic waves," US Patent 2599944A, June 10, 1952.

[5] R. L. Fante and M. T. McCormack, "Reflection properties of the Salisbury screen," IEEE Transactions on Antennas and Propagation, vol. 36, no. 10, pp. 1443-1454, 1988.

[6] B. A. Munk, Frequency Selective Surfaces, Theory and Design. New York, NY: Wiley, 2000.

[7] B. Chambers, "Optimum design of a Salisbury screen radar absorber," Electronics Letters, vol. 30, no. 16, pp. 1353-1354, 1994.

[8] B. A. Munk, P. Munk, and J. Pryor, "On designing Jaumann and circuit analog absorbers (CA Absorbers) for oblique angle of incidence," IEEE Transactions on Antennas and Propagation, vol. 55, no. 1, pp. 186-193, 2007.

[9] A. P. Sohrab and Z. Atlasbaf, "A circuit analog absorber with optimum thickness and response in X-band," IEEE Antennas and Wireless Propagation Letters, vol. 12, pp. 276279, 2013.

[10] M. Li, S. Xiao, Y. Y. Bai, and B. Z. Wang, "An ultrathin and broadband radar absorber using resistive FSS," IEEE Antennas and Wireless Propagation Letters, vol. 11, pp. 748751, 2012.

[11] G. R. Zhang, P. H. Zhou, H. B. Zhang, L. B. Zhang, J. L. $\mathrm{Xie}$, and L. J. Deng, "Analysis and design of triple-band high-impedance surface absorber with periodic diversified impedance," Journal of Applied Physics, vol. 114, article no. 164103, 2013.

[12] Y. Shang, Z. Shen, and S. Xiao, "On the design of single- 
layer circuit analog absorber using double-square-loop array," IEEE Transactions on Antennas and Propagation, vol. 61, no. 12, pp. 6022-6029, 2013.

[13] B. K. Kim and B. Lee, "Wideband absorber at X-band adopting trumpet-shaped structures," Electronics Letters, vol. 50, no. 25, pp. 1957-1959, 2014.

[14] G. Kim and B. Lee, "Design of wideband absorbers using RLC screen," Electronics Letters, vol. 51, no. 11, pp. 834836, 2015.

[15] D. Kundu, A. Mohan, and A. Chakrabarty, "Single-layer wideband microwave absorber using array of crossed dipoles," IEEE Antennas and Wireless Propagation Letters, vol. 15, pp. 1589-1592, 2016.

[16] J. Lee and B. Lee, "Wideband absorber using silver nanowire resistive film," Electronics Letters, vol. 52, no. 8, pp. 631-633, 2016.

[17] J. Lee and B. Lee, "Design of thin RC absorbers using a silver nanowire resistive screen,"," Journal of Electromagnetic Engineering and Science, vol. 16, no. 2, pp. 106-111, 2016.

[18] Y. Han, W. Che, C. Christopoulos, Y. Xiong, and Y. Chang, "A fast and efficient design method for circuit ana$\log$ absorbers consisting of resistive square-1 op arrays," IEEE Transactions on Electromagnetic Compatibility, vol. 58, no. 3, pp. 747-757, 2016.

\section{Gunyoung Kim}

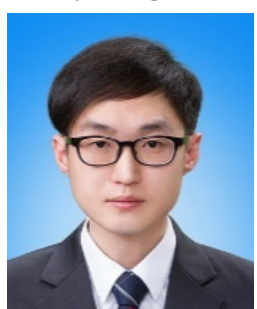

received his B.S. degree in radio communication engineering from Kyung Hee University, Yongin, Korea, in 2010 and his M.S. degree in electronics and radio engineering from Kyung Hee University in 2012. He is currently working toward his Ph.D. degree in electronics and radio engineering at the same university. His fields of research include microwave antennas, passive devices, wireless power transmission, and metamaterials.

\section{Sanghoek Kim}

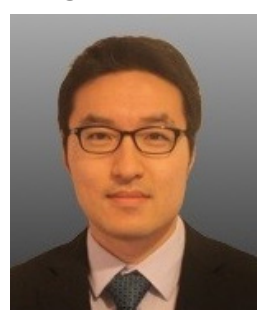

received his B.S. degree with a double major in electrical engineering and mathematics from Seoul $\mathrm{Na}^{-}$ tional University, Seoul, Korea, in 2007, and his M.S. and $\mathrm{Ph} . \mathrm{D}$. degrees in electrical engineering from Stanford University, CA, USA, in 2013. His research focuses on the applications of radio-frequency technology and electromagnetics theory in wireless power transfer, communications, and signal integrity in high-speed channels. After graduation, he worked at Qualcomm Inc., as a signal/power integrity engineer and in SiBeam Inc., as an mmWave system engineer. Currently, he is an assistant professor in the Department of Electronics and Radio Engineering at Kyung Hee University.
[19] X. Q. Lin, P. Mei, P. C. Zhang, Z. Z. D. Chen, and Y. Fan, "Development of a resistor-loaded ultrawideband absorber with antenna reciprocity," IEEE Transactions on $A n-$ tennas and Propagation, vol. 64, no. 11, pp. 4910-4913, 2016.

[20] J. Chen, Z. Hu, G. D. Wang, X. Huang, S. Wang, X. Hu, and M. Liu, "High-impedance surface-based broadband absorber with interference theory," IEEE Transactions on Antennas and Propagation, vol. 63, no. 10, pp. 4367-4374, 2015.

[21] T. H. Lee, Planar Microwave Engineering: A Practical Guide to Theory, Measurement, and Circuits. Cambridge: Cambridge University Press, 2004.

[22] M. Yoo and S. Lim, "Polarization-independent and ultrawideband metamaterial absorber using a hexagonal artificial impedance surface and a resistor-capacitor layer," IEEE Transactions on Antennas and Propagation, vol. 62, no. 5, pp. 2652-2658, 2014.

[23] S. Kim, S. Y. Kim, J. Kim, and J. H. Jung, "Highly reliable AgNW/PEDOT:PSS hybrid films: efficient methods for enhancing transparency and lowering resistance and haziness," Journal of Materials Chemistry C, vol. 2, pp. 56365643, 2014.

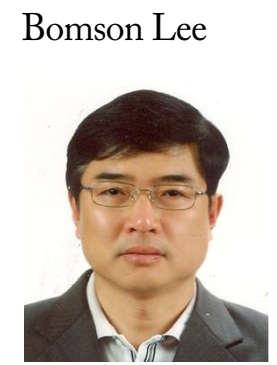

received his B.S. degree in electrical engineering from Seoul National University, Seoul, Korea, in 1982. From 1982 to 1988 , he worked at Hyundai Engineering Company Ltd., Seoul, Korea. He received his M.S. and Ph.D. degrees in electrical engineering from the University of Nebraska, Lincoln, NE, USA, in 1991 and 1995, respectively. In 1995, he joined the faculty at Kyung Hee University, where he is currently a professor in the Department of Electronics and Radio Engineering. From 2007 to 2008, he was the chair of the technical group for microwave and radio wave propagation in the Korea Institute of Electromagnetic Engineering \& Science (KIEES). In 2010, he was the editor-in-chief of the Journal of the Korean Institute of Electromagnetic Engineering and Science. From 2015 to 2016, he served as a vice chairman in KIEES. In 2017, he was an executive vice chairman in KIEES. Since 2018, he has been the president of KIEES. He was the president of KIEES in 2018. His research activities include microwave antennas, RF identification tags, microwave passive devices, wireless power transfer, and metamaterials. 


\section{APPENDIX}

For the maximum flatness of $|\Gamma(\omega)|^{2}$ at $\omega=\omega_{0}$, we require

$$
\left.\frac{d|\Gamma(\omega)|^{2}}{d \omega}\right|_{\omega=\omega_{0}}=2 G_{\Gamma} G_{\Gamma}^{\prime}+2 B_{\Gamma} B_{\Gamma}^{\prime}=0
$$

and

$$
\left.\frac{d^{2}|\Gamma(\omega)|^{2}}{d \omega^{2}}\right|_{\omega=\omega_{0}}=2\left(G_{\Gamma}^{\prime 2}+G_{\Gamma} G_{\Gamma}^{\prime \prime}+B_{\Gamma}^{\prime 2}+B_{\Gamma} B_{\Gamma}^{\prime \prime}\right)=0,
$$

where $\Gamma\left(\omega_{0}\right)=G_{\Gamma}\left(\omega_{0}\right)+j B_{\Gamma}\left(\omega_{0}\right)$, and the parameter $\omega_{0}$ is omitted for simplicity of the expression. Note that if we have both $\Gamma\left(\omega_{0}\right)=0$ and $\Gamma^{\prime}\left(\omega_{0}\right)=0$, then (A1) and (A2) are satisfied.
To satisfy $\Gamma^{\prime}\left(\omega_{0}\right)=0$, from (2), we have

$$
\frac{d \Gamma(\omega)}{d \omega}=\frac{-2 / \eta_{0}}{\left(1 / \eta_{0}+Y_{\text {in }}\right)^{2}} \cdot \frac{d Y_{\text {in }}(\omega)}{d \omega}
$$

Therefore, the choice of $L_{0}$ and $C_{0}$ to make $\frac{d Y_{i n}(\omega)}{d \omega}=0$ already helps to flatten the response by obtaining a value of zero for (A1). To further increase the flatness, we require (A2) to be satisfied. Technically, (A2) is not satisfied with $\Gamma^{\prime}\left(\omega_{0}\right)=0$ alone. However, as $\left|\Gamma\left(\omega_{0}\right)\right|$ is close to zero in any practical absorber design, (A2) is almost satisfied in practice.

Therefore, we conclude that the choice of $L_{0}$ and $C_{0}$ to make $\frac{d Y_{\text {in }}(\omega)}{d \omega}=0$ mostly satisfies both (A1) and (A2), thus maximizing the flatness of $\left|\Gamma\left(\omega_{0}\right)\right|^{2}$ at $\omega=\omega_{0}$. 